\title{
Genomics and our future food security
}

\author{
Ensuring that agricultural production meets the goal of feeding a world experiencing continued human population \\ growth and increasingly severe effects from climate change is an urgent challenge. Genomics has a role to play \\ in maximizing the utility, diversity and yield of resources, as well as in contributing to sustained food security \\ in the future.
}

eeding the world is a scientific, logistical and humanitarian enterprise involving an assembly of effort, starting with farmers and breeders and extending to policy makers and governments. The complexities of different cultural or geographic systems, combined with changing needs, technologies and environments, have made the application of broad, universal solutions for sustained food security difficult. Although the obstacles are substantial, there is a great deal of promise for increasing the efficiency and productivity of current agricultural practices. A powerful tool that we have at our disposal is genomics.

Agricultural genomics is a rich field that has been contributing to advances in crop development for decades. From sequencing reference genomes to genotyping for genome-wide association studies to genomic prediction, advances in technology and applications have led to breakthroughs in crop improvement. These innovations have resulted in elite cultivars that have been selected for agriculturally desirable traits, including high yield, stress tolerance and pest resistance. However, the environments in which crops are grown are increasingly susceptible to changing conditions in the form of unstable weather patterns or ecologies. How do we best utilize genomic technologies to adapt to this rapidly changing world?
Notably, one potential way for genomics to lend itself to crop improvement and food security is through the collectionwide sequencing and classification of established seed banks or genebanks, in which important agricultural species are stored and maintained in large collections organized by taxonomy and origin. These rich resources reflect an amazing diversity of untapped genetic potential, but there are logistical challenges to extracting the maximum information from these records. In this issue of Nature Genetics, Stein and colleagues from IPK Gatersleben use genotype-by-sequencing data from more than 22,000 barley accessions from a genebank in Germany to analyze the diversity, redundancy and relationships in this large sample. (Additional commentary can be found the accompanying News \& Views article by Langridge and Waugh.) The authors show that these 'molecular passport' data can be used to perform genome-wide association study analysis in the wellpowered dataset, thus providing insight into the selection patterns of desired traits. The large-scale genetic characterization of the entirety of this gene-bank collection provides a framework for a generalizable approach that can be applied to other crops as well.

The preservation of seed resources ensures that the natural genetic diversity captured by the collections will not be lost. Accurately cataloging these resources by using genomic data provides precise and usable information for breeders and scientists, while simplifying the effort by identifying redundancy in the collection. Expanding sequencing of other genebanks could eventually allow for a comparative and holistic view of the world-wide composition of agriculturally important crop collections, thereby providing raw material for breeding or engineering improved agricultural plant species.

The development of improved crops is a priority for scientists and policy makers alike. Indeed, one of the United Nations' Sustainable Development Goals (https:// www.un.org/sustainabledevelopment/ sustainable-development-goals/), intended to ensure a better future for all, is Zero Hunger. With the twin pressures of population growth and climate change, new strategies are needed to cope with disruptions to food growth and security, and these policies must be practical, ethical and sustainable. Outside the realm of policy, innovation is likewise needed on the scientific front. Applying genomic approaches to existing resources will greatly expand the ability to meet future challenges associated with food security, increasing human resilience in the face of a changing world-a world that should be hunger free.

Published online: 31 January 2019 https://doi.org/10.1038/s41588-019-0352-8 